\title{
Molecular Characterization Reveals Involvement of Altered El Tor Biotype Vibrio cholerae 01 Strains in Cholera Outbreak at Hyderabad, India
}

\author{
Ajay Kumar Goel ${ }^{1 *}$, Meenu Jain ${ }^{1}$, Pramod Kumar ${ }^{1}$, Pennagaram Sarguna ${ }^{2}$, \\ Meera Bai ${ }^{2}$, Neha Ghosh ${ }^{1}$, and Natrajan Gopalan ${ }^{1}$ \\ ${ }^{1}$ Biotechnology Division, Defence Research \& Development Establishment, Jhansi Road, Gwalior-474002, India \\ ${ }^{2}$ Sir Ronald Ross Institute of Tropical and Communicable Diseases, Hyderabad 500044, India
}

(Received August 16, 2010 / Accepted November 22, 2010)

\begin{abstract}
Thirty-four Vibrio cholerae isolates collected from a cholera outbreak in Hyderabad, South India were found to belong to serogroup 01 biotype El Tor serotype Ogawa. The genotype of all the isolates was confirmed by PCR assays. All the isolates were found PCR positive for $\operatorname{ctxAB}, \operatorname{omp} W, \operatorname{rfbO1}, \mathrm{rtxC}$, and $\operatorname{tcpA}$ genes. All the isolates but one harboured $r s t R^{E l ~ T o r ~}$ allele. However, one isolate carried both $r s t R^{E l}$ Tor as well as $r s t R^{\text {Classical }}$ alleles. Cholera toxin $(c t x B)$ genotyping of the isolates confirmed the presence of altered cholera toxin B of classical biotype in all the isolates. All the isolates except VCH35 harboured an RS1-CTX prophage array on the large chromosome. The isolate $\mathrm{VCH} 35$ contained a tandem repeat of classical CTX prophage on the small chromosome. The clonal relationship among the $V$. cholerae isolates as carried out by enterobacterial repetitive intergenic consensus sequences PCR, BOX PCR and randomly amplified polymorphic DNA, uniformly showed a genetic relationship among the outbreak isolates. The results of this study suggest that altered El Tor biotype $V$. cholerae with the classical cholera toxin gene are involved in cholera outbreaks in India.
\end{abstract}

Keywords: cholera, Vibrio cholerae, outbreak, cholera toxin, biotype, CTX prophage

Cholera, an acute secretory diarrhea, still remains one of the most feared infectious diseases in public health accounting for thousands of deaths annually (WHO, 2008). The disease is common in several developing countries and can be fatal in 50 percent of cases where treatment facilities are not available (Thiagarajah and Verkman, 2005). Cholera is caused by Vibrio cholerae, a Gram-negative bacterium that produces enterotoxins whose actions on the mucosal epithelium are responsible for the characteristic symptoms of the disease (Kaper et al., 1995). In the most severe cases, cholera is one of the most rapidly fatal illnesses known, with death usually resulting from severe dehydration or water loss. A healthy person may become hypotensive within an hour of the onset of symptoms and may die within two to three hours although more usually death may come within a day or so.

$V$. cholerae has more than 200 serogroups. However, strains belonging to $\mathrm{O} 1$ and $\mathrm{O} 139$ serogroup only harness the epidemic and pandemic potential. Pathogenic strains harbor a cholera toxin prophage that carries the cholera toxin (CT) genes (Waldor and Mekalanos, 1996). V. cholerae O1 strains are classified into two biotypes: classical and El Tor (Kaper et al., 1995). These two biotypes differ in their phenotypes, genotypes as well as their pathogenic potential (Safa et al., 2006). Strains belonging to El Tor biotype are supposed to have better adaptability to survive in the environment and in the human host (Finkelstein, 2006). However, the clinical manifestation of the disease caused by classical biotype strains is more severe than that caused by the El Tor strains (Kaper

* For correspondence. E-mail: akgoel73@yahoo.co.uk; Tel: +91-751-2233742; Fax: +91-751-234-1148 et al., 1995; Sack et al., 2004). The first six pandemics were caused by classical biotype but after $1961 \mathrm{~V}$. cholerae El Tor biotype displaced classical biotype (Kaper et al., 1995). There are certain structural and functional peculiarities in the virulence genes of El Tor vibrios that make the symptoms of cholera milder but more long lasting than classical biotype strains (Smirnova et al., 2004). However, in the recent past, $V$. cholerae strains having phenotypic and genotypic traits of both biotypes have been noticed (Nair et al., 2006; Goel et al., 2008; Goel and Jiang, 2010). These new variants which cannot be biotyped as classical or El Tor and harbour the classical type cholera toxin $(\operatorname{ctx} B)$ gene have been termed as "atypical El Tor" strains (Safa et al., 2010).

In May 2009, hundreds of people were affected in a suddenly erupted cholera outbreak in the Bholakpur area, Hyderabad, South India. In this study, we report the genetic analysis and molecular typing of $V$. cholerae El Tor strains isolated from the patients of this outbreak.

\section{Materials and Methods}

Bacterial strains

A total of $34 \mathrm{~V}$. cholerae $\mathrm{O} 1$ strains were analysed in this study. All the $V$. cholerae $\mathrm{O} 1 \mathrm{El}$ Tor strains were isolated from clinical cases during the cholera outbreak in Hyderabad as described earlier (Pourshafie et al., 2007). Other bacterial strains used in the study were $V$. cholerae O1 (ATCC 11623), V. cholerae O1 (ATCC 14033) and V. cholerae $\mathrm{O} 139$.

Biochemical and serological characterization All the bacterial isolates were screened for oxidase reaction followed 
Table 1. PCR primers used in this study

\begin{tabular}{|c|c|c|}
\hline Target gene & Forward primer & Reference \\
\hline ompWF & CACCAAGAAGGTGACTTTATTGTG & Nandi et al. (2000) \\
\hline ompWR & GGTTTGTCGAATTAGCTTCACC & Nandi et al. (2000) \\
\hline$c t x A B F$ & GCCGGGTTGTGGGAATGCTCCAAG & Goel et al. (2007) \\
\hline$c t x A B R$ & CATGCGATTGCCGCAATTAGTATGGC & Goel et al. (2007) \\
\hline$r f b O 1 F$ & TCTATGTGCTGCGATTGGTG & Goel et al. (2007) \\
\hline$r f b O 1 R$ & CCCCGAAAACCTAATGTGAG & Goel et al. (2007) \\
\hline tcp $A F$ & CGTTGGCGGTCAGTCTTG & Goel et al. (2007) \\
\hline $\operatorname{tcp} A R$ & CGGGCTTTCTTCTTGTTCG & Goel et al. (2007) \\
\hline$r t x C F$ & CGACGAAGATCATTGACGAC & Chow et al. (2001) \\
\hline $\mathrm{rtxCF}$ & CATCGTCGTTATGTGGTTGC & Chow et al. (2001) \\
\hline$r s t R^{E l T o r}$ & СТTCTCATCAGCAAAGCCTCCATC & Bhattacharya et al. (2006) \\
\hline$r s t R^{\text {Clasical }}$ & GCACCATGATTTAAGATGCTC & Bhattacharya et al. (2006) \\
\hline$r s t R^{\text {Calcutta }}$ & CTGTAAATCTCTTCAATCCTAGG & Bhattacharya et al. (2006) \\
\hline$r s t R R$ & TCGAGTTGTAATTCATCAAGAGTG & Bhattacharya et al. (2006) \\
\hline rstCF & GATGTTTACGATAGCCTAGAAGACTT & Nguyen et al. (2009) \\
\hline$r s t C R$ & TACAGTGATGGCTCAGTCAATGC & Nguyen et al. (2009) \\
\hline $\operatorname{ch} 1 F$ & GACCACTCAGGCCGCTGAAAT & Nguyen et al. (2009) \\
\hline $\operatorname{ch} 1 R$ & CCGCGCTCAAGTGGTTATCGG & Nguyen et al. (2009) \\
\hline $\operatorname{ctx} B F$ & AGATATTTTCGTATACAGAATCTCTAG & Nguyen et al. (2009) \\
\hline cepR & AAACAGCAAGAAAACCCCGAGT & Nguyen et al. (2009) \\
\hline rstAR & CCGTGAAAGTCATCAACG & Nguyen et al. (2009) \\
\hline $\operatorname{ch} 2 F$ & AACAACAGGTTGCAAGAGAGCATT & Nguyen et al. (2009) \\
\hline $\operatorname{ch} 2 R$ & TATTGCTTTTTTAATGGCCGTT & Nguyen et al. (2009) \\
\hline
\end{tabular}

by standard biochemical tests for presumptive identification of $V$. cholerae (Nair et al., 1987; Tamrakar et al., 2006). Serological identification of the isolates was done by slide agglutination using commercially available polyvalent antiserum against Vibrio cholerae $\mathrm{O} 1$ (Ogawa and Inaba) and O139 serogroups (Difco, USA).

\section{DNA preparation}

DNA was extracted by using the Fermentas genomic DNA extraction kit as per the manufacturer instructions (MBI Fermentas, Lithuania). The amount and purity of the DNA was measured by spectrophotometer (NanoDrop ND-1000, Australia).

\section{Gene detection by multiplex PCR}

Various toxigenic and pathogenic genes were detected by PCR as described elsewhere (Kumar et al., 2009). The genes encoding cholera toxin $(c t x A B)$, an outer membrane protein $(o m p W)$, O1 somatic antigen $(r f b O 1)$, repeat in toxin $(r t x C)$ and toxin coregulated pilus $(t c p A)$ were detected by PCR assay. Isolates were subjected to $r s t R$ typing using the primers for $r s t R^{E l ~ T o r}, r s t R^{\text {Classical }}$, and $r s t R^{\text {Calcuta }}$ as described elsewhere (Bhattacharya et al., 2006). A list of all primers used in this study has been given in Table 1 . The amplicons were electrophoresed through $1.2 \%$ agarose gel and then stained with ethidium bromide.

\section{Sequence of $c t x B$ gene}

The cholera toxin B (ctxB) gene was amplified from the strains isolated from outbreak areas using the $c t x F$ and $c t x R$ primers as reported earlier (Olsvik et al., 1993). The PCR product was purified and sequencing was carried out using the same primers on a 96 capillary model 3730xl system by using the Big Dye Terminator kit from Applied Biosystems. The sequences of $c t x B$ gene for other $V$ cholerae $\mathrm{O} 1 \mathrm{El}$ Tor and classical strains were retrieved from the public domain
(GenBank). The deduced amino acid sequences of $\operatorname{ctx} B$ gene from all the strains were aligned using the CLUSTAL $\mathrm{W}$ programme. The GenBank accession no. for the $[c t x B]$ sequence of $\mathrm{VCH} 2, \mathrm{VCH} 4$, VCH5, VCH8, VCH10, VCH12. VCH16, VCH19, VCH22, VCH26, VCH31, and VCH34 are GU569396, GU569397, GU569398, GU 569399, GU569400, GU569401, GU569402, GU569403, GU569404, GU569405, GU569406, and GU569407, respectively.

\section{Presence and location of RS1 and CTX prophage}

The presence of the RS1 element was confirmed by PCR using the $r s t C$ specific primers $r s t C F / r s t C R$ as described elsewhere (Nguyen $e t$ al., 2009). The location of an RS1-CTX prophage array on the large chromosome was determined by using two sets of primers, Ch1F/rstAR and $c t x B F / C h 1 R$ (Nguyen et al., 2009). The absence of CTX prophage or RS1 on the small chromosome was established using primers $\operatorname{ch} 2 F / \operatorname{ch} 2 R$. Another set of primers, $\operatorname{ch} 2 F / r s t A R$ was used to determine the presence of tandem repeat of the CTX prophage on the small chromosome.

\section{Genomic fingerprinting}

Representative isolates were examined for clonal relationship using the enterobacterial repetitive intergenic consensus sequence (ERIC) PCR, BOX PCR, and RAPD. ERIC PCR was performed as described earlier with little modifications by using two primers ERIC1R 5'-ATGTAAGCTCCTGGGGATTCAC-3' and ERIC2 5'-AAGTAAG TGACTGGGTGAGCG-3' (Rivera et al., 1995). The thermal cycler was programmed for 35 cycles of $1 \mathrm{~min}$ at $94^{\circ} \mathrm{C}, 1 \mathrm{~min}$ at $52^{\circ} \mathrm{C}, 10$ min at $68^{\circ} \mathrm{C}$ followed by $20 \mathrm{~min}$ incubation at $70^{\circ} \mathrm{C}$. The BOX PCR was performed by using a single nucleotide primer BOX A1R (Versalovic et al., 1994). BOX PCR was performed by using a single nucleotide primer BOX A1R 5'-CTACGGCAAGGCGACGCTGAC G-3' (Versalovic et al., 1994). PCR program consisted of: initial dena- 


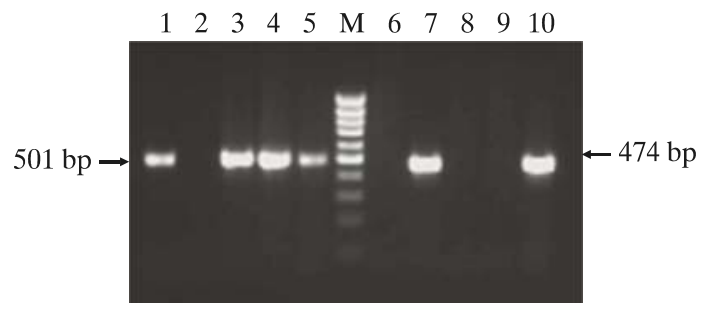

Fig. 1. $r s t R$ typing of $V$. cholerae outbreak isolates. Lanes: M, 100 bp ladder; $1-5$, rst $R^{E l ~ T o r} ; 6-10$, rst $R^{\text {Classical }}$. Lanes: 1 and $6, V$. cholerae O1 ATCC 14033; 2 and 7, V. cholerae O1 ATCC 11623; 3 and 8, V. cholerae $\mathrm{VCH} 4 ; 4$ and $9, \mathrm{~V}$. cholerae $\mathrm{VCH} 20 ; 5$ and $10, V$. cholerae $\mathrm{VCH} 35$.

turation $\left(95^{\circ} \mathrm{C}\right.$ for $\left.7 \mathrm{~min}\right), 30$ cycles of $94^{\circ} \mathrm{C}$ for $1 \mathrm{~min}, 53^{\circ} \mathrm{C}$ for $1 \mathrm{~min}, 65^{\circ} \mathrm{C}$ for $8 \mathrm{~min}$, and a final extension of $65^{\circ} \mathrm{C}$ for $16 \mathrm{~min}$. RAPD was performed using 10-mer random primer from Operon 10-mer kit A (Operon Technologies, USA). The PCR reaction consisted of $20 \mathrm{ng}$ of DNA, $20 \mathrm{pmol}$ of primer in Taq buffer $2.5 \mathrm{mmol} / \mathrm{L}$ $\mathrm{MgCl}_{2}, 200 \mu \mathrm{mol} / \mathrm{L}$ of each $\mathrm{dNTP}$ and $1 \mathrm{U}$ of Taq polymerase in a final volume of $25 \mu \mathrm{l}$. The PCR cycling conditions were as follows: preincubation at $94^{\circ} \mathrm{C}$ for $2 \mathrm{~min}, 40$ cycles of $1 \mathrm{~min}$ at $94^{\circ} \mathrm{C}, 1 \mathrm{~min}$ at $36^{\circ} \mathrm{C}$ and $2 \mathrm{~min}$ at $72^{\circ} \mathrm{C}$, and a final incubation for $5 \mathrm{~min}$ at $72^{\circ} \mathrm{C}$.

\section{Results and Discussion}

A total of $34 \mathrm{~V}$. cholerae were isolated from the affected patients during the outbreak. All the isolates were biochemically identified as $V$. cholerae and serologically confirmed as $\mathrm{O} 1$ Ogawa.

\section{Genotype by PCR}

The PCR analysis of the isolates confirmed the presence of $c t x A B$, omp $W, r f b O 1, r t x C$, and $t c p A$ genes. The omp $W$ gene is species-specific for $V$. cholerae and its presence in the isolates confirmed the $V$. cholerae. The presence of $\operatorname{ctx} A B$ and $r f b O 1$ genes confirmed the toxigenicity and group 1 antigen of the "O" side chain of the LPS of $V$. cholerae. Biotype of $V$. cholerae $\mathrm{O} 1$ serogroup isolates can be determined on the basis of PCR assays developed for $r t x A$ or $r t x C$ (Chow et al., 2001). In this study, all the isolates belonged to the El Tor biotype on the basis of the repeat in toxin gene $(r t x C)$.
Cholera toxin is one of the most important epidemic marker among various toxins produced by $V$. cholerae (Kaper et al., 1995). The cholera toxin gene $(\operatorname{ctx} A B)$ is acquired from the genome of a filamentous CTX bacteriophage. The pilus colonization factor TCP acts as a receptor for CTX $\varnothing$, which can infect non-toxigenic $V$. cholerae, leading to the emergence of new toxigenic strains (Waldor and Mekalanos, 1996).

\section{Rst $R$ typing}

PCR analysis for $r s t R$ allele in the isolates revealed that the isolates were of $r s t R^{E l}$ Tor type (Fig. 1). Only one isolate (VCH35) was found positive for $r s t R^{E l}$ Tor as well as $r s t R^{\text {Classical }}$ genes indicating the presence of genes from both, the El Tor as well as classical CTX phage. The rst $R$ alleles are believed to be biotype specific and are classified into $r s t R^{\text {Classical }}, r s t R^{\mathrm{El} \text { Tor }}$, and $r s t R^{\text {Calcutta }}$ for classical, El Tor, and O139 alleles, respectively (Kimsey et al. 1998). However, presence of both $r s t R^{\mathrm{EI} T o r}$ and $r s t R^{\text {Classical }}$ allele in the same strain indicates the presence of two different copies of CTХФ, either as a tandem array or located on a different chromosome (Nguyen et al., 2009). Likewise, multiple $r s t R$ alleles in the same $V$. cholerae strain have been reported from other countries (Kimsey et al., 1998; Nusrin et al., 2004).

\section{Sequence of $\operatorname{ctx} B$ gene}

The alignment of $c t x B$ sequences from outbreak strains with $\mathrm{O} 1 \mathrm{El}$ Tor and $\mathrm{O} 1$ classical reference strains revealed that the $\operatorname{ctx} B$ gene sequences of the isolates were identical and were aligned with that of the classical biotype of $\operatorname{ctx} B$. The deduced amino acid sequences of CT-B from representative El Tor isolates of $V$. cholerae varied from CT-B of reference El Tor strains at position 39 (histidine in place of tyrosine) and 68 (threonine in place of isoleucine). Similar El Tor strains producing classical CT were isolated from many parts of Asian countries (Ansaruzzaman et al., 2004; Nair et al., 2006; Nguyen et al., 2009; Goel and Jiang, 2010). Biotype specific СТХ $\Phi$ is found in the $V$. cholerae strains. El Tor biotype strains harbour $\mathrm{CTX}^{\mathrm{El} \text { Tor }} \Phi$ and classical strains have $\mathrm{CTX}^{\text {Classical }} \Phi$ (Ansaruzzaman et al., 2004). However, in this study $V$. cholerae $\mathrm{O} 1 \mathrm{El}$ Tor isolates have shed their El Tor CTX prophage and acquired the classical prophage. Therefore, all the El Tor isolates were found to possess the $\operatorname{ctx} B$ sequence of the classical biotype. Classical biotype strains were replaced by the El Tor biotype in the seventh and current pandemic

(A)

(B)

(C)

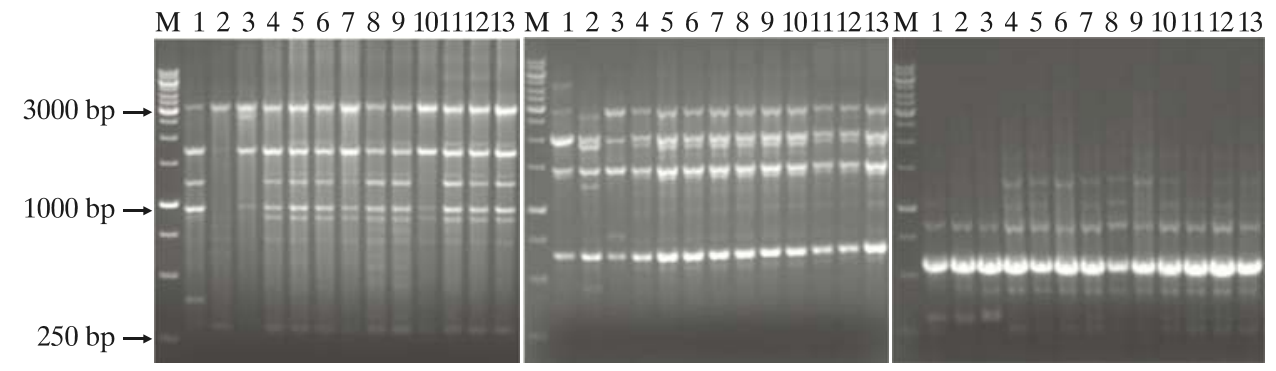

Fig. 2. DNA fingerprints of different outbreak isolates of $V$. cholerae O1 generated by ERIC PCR (A), Box PCR (B), and RAPD PCR (C). Lanes: M, Ladder; 1, V. cholerae O1 ATCC 11623; 2, V. cholerae O1 ATCC 14033; 3, V. cholerae O139; 4-13, outbreak isolates: 4, VCH2; 5, VCH4; 6, VCH5; 7, VCH8; 8, VCH10; 9, VCH12; 10, VCH16; 11, VCH19; 12, VCH22; $13, \mathrm{VCH} 34$. 
of cholera. Both biotypes of $V$. cholerae $\mathrm{O} 1$ are closely related in their $\mathrm{O}$-antigen biosynthetic genes. However, the genomic structure of the СТХФ, in which the cholera toxin genes are contained, differs between the classical and El Tor biotypes (Waldor and Mekalanos, 1996; Ansaruzzaman et al., 2004).

\section{RS1 element and CTX prophage array}

The $r s t C$ specific primers amplified the DNA fragments from all the isolates indicating the presence of the $r s t C$ gene in the chromosome. The PCR amplified products using $c h 1 F /$ rstAR and $c t x B F / c h 1 R$ primers in all the isolates confirmed that the RS1-CTX prophage array is located on the large chromosome. All the isolates yielded PCR products using $\operatorname{ch} 2 \mathrm{~F} / \mathrm{ch} 2 \mathrm{R}$ primers indicating the absence of the CTX prophage or RS1 element on small chromosome. However, the isolate VCH35 was PCR negative for $c h 2 F / c h 2 R$ primers indicating the presence of the CTX prophage or RS1 element on the small chromosome. Further, VCH35 was PCR positive using $c h 2 F / r s t A R$ primers confirming the presence of tandem repeat of the CTX prophage on the small chromosome. Atypical El Tor strains have been classified into two different groups on the basis of genetic structures of the CTX prophage and RS1 element on each chromosome (Lee et al., 2009; Nguyen et al., 2009). Group 1 strains harbor a tandem repeat of the classical CTX prophage on the small chromosome and Group II strains contain the RS1 and CTX prophage with $\mathrm{El}$ Tor type $r s t R$ and classical $c t x B$ on the large chromosome (Lee et al., 2009; Choi et al., 2010). All the strains used in this study belonged to Group II strains. However, the isolate VCH35 seemed to belong to Group 1 strains.

\section{DNA fingerprinting}

Fingerprinting analysis by ERIC PCR, BOX PCR, and RAPD PCR revealed the clonal relationships among all the outbreak isolates. ERIC-PCR with genomic DNA of various $V$. cholerae strains resulted in amplification of multiple fragments of DNA in sizes ranging between 0.25 and $3.2 \mathrm{~kb}$ (Fig. 2A). The BOX PCR of genomic DNA from various $V$. cholerae isolates also resulted in amplification of different fragments of DNA of varying length ranging from $0.65 \mathrm{~kb}$ to $3.0 \mathrm{~kb}$ (Fig. 2B). Likewise, different DNA fragments of the same size were amplified by RAPD in all the isolates (Fig. 2C). However, in the entire outbreak $V$. cholerae isolates, fragments of similar sizes were found. PCR-based methods of fingerprinting take advantage of the presence of repetitive sequences that are interspersed throughout the genome of diverse bacterial species. The same genetic pattern was found among all the isolates using all three fingerprinting techniques suggesting the outbreak is likely caused by a single clone of $V$. cholerae O1 strain with altered biotype. Similar $V$. cholerae strains have been isolated from other cholera outbreaks in India (Kumar et al., 2009; Goel et al., 2010).

In conclusion, the Vibrio cholerae O1 'atypical El Tor' biotype strains with $r s t R^{\mathrm{El} T o r}$ allele were involved in the Hyderabad cholera outbreak. Most of the isolates contained an RS1-CTX prophage array on the large chromosome and harboured the classical cholera toxin $(c t x B)$ gene. The results of this study showed that classical CT producing El Tor strains are now replacing the seventh pandemic El Tor strains. This is an evo- lutionary optimization of the El Tor biotype, which could represent a new and more significant emerging form of the El Tor biotype of $V$. cholerae.

\section{Acknowledgements}

The authors thank Director, DRDE, Gwalior for providing necessary facilities and financial support for the work.

\section{References}

Ansaruzzaman, M., N.A. Bhuiyan, G.B. Nair, D.A. Sack, M. Lucas, J.L. Deen, J. Ampuero, and C.L. Chaignat. 2004. Cholera in Mozambique, variant of Vibrio cholerae. Emerg. Infect. Dis. 10, 2057-2059.

Bhattacharya, T., S. Chatterjee, D. Maiti, R.K. Bhadra, Y. Takeda, G.B. Nair, and R.K. Nandy. 2006. Molecular analysis of the $r s t R$ and orfU genes of the CTX prophages integrated in the small chromosomes of environmental Vibrio cholerae non-O1, nonO139 strains. Environ. Microbiol. 8, 526-634.

Choi, S.Y., J.H. Lee, Y.S. Jeon, H.R. Lee, E.J. Kim, M. Ansaruzzaman, N.A. Bhuiyan, and et al. 2010. Multilocus variable-number tandem repeat analysis of Vibrio cholerae O1 El Tor strains harbouring classical toxin B. J. Med. Microbiol. 59, 763-769.

Chow, K.H., T.K. Ng, K.Y. Yuen, and W.C. Yam. 2001. Detection of RTX toxin gene in Vibrio cholerae by PCR. J. Clin. Microbiol. 39, 2594-2597.

Finkelstein, R.A. 2006. Vibrio cholerae O1 and O139, and other pathogenic vibrios. [Online] http://gsbs.utmb.edu/microbook/ch024.htm.

Goel, A.K., M. Jain, P. Kumar, S. Bhadauria, D.V. Kmboj, and L. Singh. 2008. A new variant of Vibrio cholerae O1 El Tor causing cholera in India. J. Infect. 57, 280-281.

Goel, A.K., M. Jain, P. Kumar, and S.C. Jiang. 2010. Molecular characterization of Vibrio cholerae outbreak strains with altered El Tor biotype from southern India. World J. Microbiol. Biotechnol. 26, 281-287.

Goel, A.K. and S.C. Jiang. 2010. Genetic determinants of virulence, antibiogram and altered biotype among the Vibrio cholerae O1 isolates from different cholera outbreaks in India. Infect. Genet. Evol. 10, 815-819.

Goel, A.K., S. Ponmariappan, D.V. Kamboj, and L. Singh. 2007. Single multiplex polymerase chain reaction for environmental surveillance of toxigenic-pathogenic O1 and non-O1 Vibrio cholerae. Folia Microbiol (Praha). 52, 81-85.

Kaper, J.B., J.G. Morris, Jr., and M.M. Levine. 1995. Cholera. Clin. Microbiol. Rev. 8, 48-86.

Kimsey, H.H., G.B. Nair, A. Ghosh, and M.K. Waldor. 1998. Diverse CTXphis and evolution of new pathogenic Vibrio cholerae. Lancet 352, 457-458.

Kumar, P., M. Jain, A.K. Goel, S. Bhadauria, S.K. Sharma, D.V. Kamboj, L. Singh, T. Ramamurthy, and G.B. Nair. 2009. A large cholera outbreak due to a new cholera toxin variant of the Vibrio cholerae $\mathrm{O} 1 \mathrm{El}$ Tor biotype in Orissa, Eastern India. J. Med. Microbiol. 58, 234-238.

Lee, J.H., S.Y. Choi, Y.S. Jeon, H.R. Lee, E.J. Kim, B.M. Nguyen, N.T. Hien, and et al. 2009. Classification of hybrid and altered Vibrio cholerae strains by CTX prophage and RS1 element structure. J. Microbiol. 47, 783-788.

Nair, G.B., S. Misra, R.K. Bhadra, and S.C. Pal. 1987. Evaluation of the multitest medium for rapid presumptive identification of Vibrio cholerae from environmental sources. Appl. Environ. Microbiol. 53, 1203-1205.

Nair, G.B., F. Qadri, J. Holmgren, A.M. Svennerholm, A. Safa, N.A. Bhuiyan, Q.S. Ahmad, and et al. 2006. Cholera due to altered El Tor strains of Vibrio cholerae O1 in Bangladesh. J. Clin. Microbiol. 44, 4211-4213. 
Nandi, B., R.K. Nandy, S. Mukhopadhyay, G.B. Nair, T. Shimada, and A.C. Ghose. 2000. Rapid method for species-specific identification of Vibrio cholerae using primers targeted to the gene of outer membrane protein OmpW. J. Clin. Microbiol. 38, 4145-4151.

Nguyen, B.M., J.H. Lee, N.T. Cuong, S.Y. Choi, N.T. Hien, D.D. Anh, H.R. Lee, and et al. 2009. Cholera outbreaks caused by an altered Vibrio cholerae O1 El Tor biotype strain producing classical cholera toxin B in Vietnam in 2007 to 2008. J. Clin. Microbiol. 47, 1568-1571.

Nusrin, S., G.Y. Khan, N.A. Bhuiyan, M. Ansaruzzaman, M.A. Hossain, A. Safa, R. Khan, and et al. 2004. Diverse CTX phages among toxigenic Vibrio cholerae O1 and O139 strains isolated between 1994 and 2002 in an area where cholera is endemic in Bangladesh. J. Clin. Microbiol. 42, 5854-5856.

Olsvik, O., J. Wahlberg, B. Petterson, M. Uhlen, T. Popovic, I.K. Wachsmuth, and P.I. Fields. 1993. Use of automated sequencing of polymerase chain reaction-generated amplicons to identify three types of cholera toxin subunit $\mathrm{B}$ in Vibrio cholerae $\mathrm{O} 1$ strains. J. Clin. Microbiol. 31, 22-25.

Pourshafie, M.R., B. Bakhshi, R. Ranjbar, M. Sedaghat, N. Sadeghifard, J. Zaemi Yazdi, M. Parzadeh, and J. Raesi. 2007. Dissemination of a single Vibrio cholerae clone in cholera outbreaks during 2005 in Iran. J. Med. Microbiol. 56, 1615-1619.

Rivera, I.G., M.A. Chowdhury, A. Huq, D. Jacobs, M.T. Martins, and R.R. Colwell. 1995. Enterobacterial repetitive intergenic consensus sequences and the PCR to generate fingerprints of genomic DNAs from Vibrio cholerae O1, O139, and non-O1 strains. Appl. Environ. Microbiol. 61, 2898-2904.

Sack, D.A., R.B. Sack, G.B. Nair, and A.K. Siddique. 2004. Cholera. Lancet 363, 223-233.

Safa, A., N.A. Bhuyian, S. Nusrin, M. Ansaruzzaman, M. Alam, T. Hamabata, Y. Takeda, D.A. Sack, and G.B. Nair. 2006. Genetic characteristics of Matlab variants of Vibrio cholerae $\mathrm{O} 1$ that are hybrids between classical and El Tor biotypes. J. Med. Microbiol. 55, 1563-1569.

Safa, A., G.B. Nair, and R.Y. Kong. 2010. Evolution of new variants of Vibrio cholerae O1. Trends Microbiol. 18, 46-54.

Smirnova, N.I., N.B. Cheldyshova, S.P. Zadnova, and V.V. Kutyrev. 2004. Molecular-genetic peculiarities of classical biotype Vibrio cholerae, the etiological agent of the last outbreak Asiatic cholera in Russia. Microb. Pathog. 36, 131-139.

Tamrakar, A.K., A.K. Goel, D.V. Kamboj, and L. Singh. 2006. Surveillance methodology for Vibrio cholerae in environmental samples. Int. J. Environ. Health Res. 16, 305-312.

Thiagarajah, J.R. and A.S. Verkman. 2005. New drug targets for cholera therapy. Trends Pharmacol. Sci. 26, 172-175.

Versalovic, J., M. Schneider, F.J.d. Brulin, and J.R. Lupski. 1994. Genomic fingerprinting of bacteria using repetitive sequence-based polymerase chain reaction. Methods Mol. Cell. Biol. 5, 25-40.

Waldor, M.K. and J.J. Mekalanos. 1996. Lysogenic conversion by a filamentous phage encoding cholera toxin. Science 272, 19101914.

WHO. 2008. Cholera, 2007. Wkly Epidemiol Rec. 83, 269-283. 